TRANSACTIONS OF THE

AMERICAN MATHEMATICAL SOCIETY

Volume 357, Number 2, Pages 509-534

S 0002-9947(04)03495-6

Article electronically published on September 2, 2004

\title{
DISCRETE MORSE FUNCTIONS FROM LEXICOGRAPHIC ORDERS
}

\author{
ERIC BABSON AND PATRICIA HERSH
}

\begin{abstract}
This paper shows how to construct a discrete Morse function with a relatively small number of critical cells for the order complex of any finite poset with $\hat{0}$ and $\hat{1}$ from any lexicographic order on its maximal chains. Specifically, if we attach facets according to the lexicographic order on maximal chains, then each facet contributes at most one new face which is critical, and at most one Betti number changes; facets which do not change the homotopy type also do not contribute any critical faces. Dimensions of critical faces as well as a description of which facet attachments change the homotopy type are provided in terms of interval systems associated to the facets. As one application, the Möbius function may be computed as the alternating sum of Morse numbers.

The above construction enables us to prove that the poset $\Pi_{n} / S_{\lambda}$ of partitions of a set $\left\{1^{\lambda_{1}}, \ldots, k^{\lambda_{k}}\right\}$ with repetition is homotopy equivalent to a wedge of spheres of top dimension when $\lambda$ is a hook-shaped partition; it is likely that the proof may be extended to a larger class of $\lambda$ and perhaps to all $\lambda$, despite a result of Ziegler (1986) which shows that $\Pi_{n} / S_{\lambda}$ is not always Cohen-Macaulay.
\end{abstract}

\section{INTRODUCTION}

Let us begin by reviewing the notion of a discrete Morse function, as established by Robin Forman in [13]. A function $f$ which assigns real values to the cells in a CW-complex is a discrete Morse function if (1) for each $d$-cell $\sigma$, denoted $\sigma^{(d)}$, the sets $\left\{\tau^{(d-1)} \subseteq \bar{\sigma} \mid f\left(\tau^{(d-1)}\right) \geq f(\sigma)\right\}$ and $\left\{\overline{\tau^{(d+1)}} \supseteq \sigma \mid f\left(\tau^{(d+1)}\right) \leq f(\sigma)\right\}$ each have cardinality at most one, and (2) $f(\sigma) \geq f(\tau)$ for $\sigma^{(d)} \subseteq \overline{\tau^{(d+1)}}$ implies that $\sigma$ is a regular face of $\tau$. Requiring condition (1) for every $\sigma$ implies that for each $\sigma$, at most one of these cardinalities is greater than 0 ; when both cardinalities are 0 , then $\sigma$ is called a critical cell. Figure 1 gives an example of a discrete Morse function on a 1-sphere resulting from a height function.

Critical cells record changes in topological structure as a complex is built by sequentially inserting cells in the order specified by the Morse function, with critical $i$-cells behaving similarly to critical points of index $i$ in a traditional Morse function; the noncritical cells may be eliminated by elementary collapses without changing the homotopy type. This collapsing results from the fact that noncritical cells come

Received by the editors July 1, 2003.

2000 Mathematics Subject Classification. Primary 05E25; Secondary 05A17, 05A18, 55P15.

Key words and phrases. Discrete Morse theory, poset, order complex, partition. 


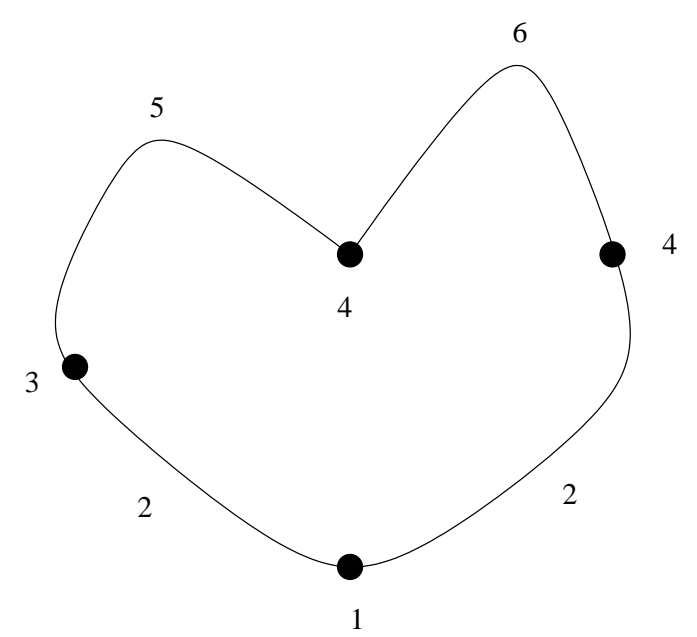

Figure 1. A discrete Morse function

in pairs $\sigma^{(p)} \subseteq \overline{\tau^{(p+1)}}$ which prevent each other from being critical by satisfying $f(\sigma) \geq f(\tau)$. Thus, a discrete Morse function $f$ gives rise to a matching on the noncritical cells in the face poset, namely in the partial order on cells defined by letting $\sigma$ be less than $\tau$ for each $\sigma$ in the boundary of $\tau$.

Definition 1.1. A matching is acyclic if the directed graph obtained by directing face poset edges belonging to the matching upwards and directing all other poset edges downward has no directed cycles.

Observe that the face poset matching resulting from a discrete Morse function is always acyclic, since the edges are oriented in the direction in which $f$ decreases. Conversely, many different (but in some sense equivalent) discrete Morse functions may be constructed from any face poset acyclic matching. The unmatched cells in an acyclic matching are exactly the critical cells of any corresponding discrete Morse function. We shall work primarily in terms of acyclic matchings, but with this correspondence (due to Chari [11) always in the background.

Let us deviate slightly from Forman's conventions by including the empty set in the domain of $f$ and in the face posets upon which we construct acyclic matchings; this has the effect of expressing results in terms of reduced Euler characteristic and reduced homology. Let $m_{i}$ be the number of critical cells of dimension $i$ in a discrete Morse function, let $b_{i}$ be the Betti number recording the rank of $H_{i}(\Delta)$, and let $M_{a}$ be the partial complex comprised of those cells $\sigma$ such that $f(\sigma) \leq a$. When using the reduced version of discrete Morse theory, we denote the number of critical $i$-cells by $\tilde{m}_{i}$ and the reduced Betti numbers by $\tilde{b}_{i}$. It is shown in 13 that $\Delta$ collapses onto a complex $\Delta^{M}$ comprised of the discrete Morse function critical cells in a way that preserves homotopy type. Specifically, Forman shows that the following results carry over from traditional Morse theory, the first two of which are called the Morse inequalities.

(1) $\tilde{m}_{j} \geq \tilde{b}_{j}$ for $-1 \leq j \leq \operatorname{dim}(\Delta)$.

(2) $\sum_{i=0}^{j+1}(-1)^{i} \tilde{m}_{j-i} \geq \sum_{i=0}^{j+1}(-1)^{i} \tilde{b}_{j-i}$ for $0 \leq j \leq \operatorname{dim}(\Delta)$, with equality achieved when $j=\operatorname{dim}(\Delta)$. 
(3) If there are no critical cells $\sigma$ satisfying $a \leq f(\sigma) \leq b$ for some $a<b$, then the partial complex $M_{b}$ collapses onto the partial complex $M_{a}$. In particular, $\Delta$ is homotopy equivalent to the complex resulting from a series of such collapses.

From these facts, it immediately follows that:

(1) If $\tilde{m}_{i}=0$ for all $i$, then $\Delta$ is collapsible.

(2) If $\tilde{m}_{i}=0$ for all $i \neq j$ for some fixed $j$, then $\Delta$ is homotopy equivalent to a wedge of $j$-spheres.

Figure 1 gives an example of a (nonreduced) discrete Morse function with $b_{0}=$ $b_{1}=1$ and $m_{0}=m_{1}=2$. Letting $f(\emptyset)=1.5$ turns this into a reduced discrete Morse function with $\tilde{b}_{0}=0, \tilde{m}_{0}=\tilde{b}_{1}=1$ and $\tilde{m}_{1}=2$. The Morse numbers are larger than the Betti numbers because there is a critical cell of dimension 0 that is labeled 4 which locally looks as though it is creating a new connected component as the complex is built from bottom to top, and there is a critical cell of dimension one that is labeled 5 which locally appears to be closing off a 1-cycle, but these two critical cells actually cancel each other's effect. Forman notes that whenever an acyclic matching has two critical cells $\sigma^{(p)}$ and $\tau^{(p+1)}$ such that there is a unique gradient path from $\tau$ to $\sigma$ (i.e. a path upon which $f$ decreases at each step), then one may obtain a new acyclic matching in which $\sigma$ and $\tau$ are no longer critical by reversing this gradient path, since doing so cannot create any directed cycles. Such a reversal for instance straightens our 1-sphere into a standard circle, which only has one critical 0-cell and a single critical 1-cell. This mechanism for cancelling critical cells will be particularly useful in conjunction with "lexicographic discrete Morse functions" because they are well-suited to verifying uniqueness of gradient paths, as demonstrated in the application of Section 5.

We shall consider finite posets with unique minimal and maximal elements $\hat{0}$ and $\hat{1}$. A chain $\hat{0}=u_{0}<u_{1}<\cdots<u_{r}=\hat{1}$ of comparable poset elements is saturated (or maximal) if $u_{i} \leq v \leq u_{i+1}$ implies $v=u_{i}$ or $v=u_{i+1}$ for $0 \leq i<r$. A poset $P$ is graded if for each $x \in P$, all saturated chains from $\hat{0}$ to $x$ have the same length, called the rank of $x$. We will not require our posets to be graded, but we will refer to the rank of an element within a saturated chain, not requiring this to agree with its rank within other saturated chains.

Recall that the order complex, denoted $\Delta(P)$, for a finite poset $P$ with $\hat{0}$ and $\hat{1}$ is the simplicial complex comprised of an $i$-face for each chain $\hat{0}<v_{0}<\cdots<v_{i}<\hat{1}$ of $i+1$ comparable poset elements. For instance, the order complex of the face poset of any simplicial complex $K$ is the first barycentric subdivision of $K$, allowing one to apply poset methods to arbitrary simplicial complexes (for example, Kozlov uses this approach on certain graph complexes in [19]). Figure 2 provides an example of a poset and its order complex. Our discrete Morse function will use an ordering on facets of $\Delta(P)$, i.e. on saturated chains in $P$.

The Möbius function of a finite poset $P$ is a function on pairs of elements, defined as follows: $\mu_{P}(u, u)=1$ and $\mu_{P}(u, w)=-\sum_{u<v<w} \mu_{P}(u, v)$; Möbius functions arise in combinatorics as the coefficients in inclusion-exclusion counting formulas. The Möbius function of $P$ satisfies $\mu_{P}(\hat{0}, \hat{1})=\tilde{\chi}(\Delta(P))$ (cf. [20]), so our discrete Morse functions for poset order complexes will give a way to compute $\mu_{P}(x, y)$ as the alternating sum of Morse numbers on the order complex restriction $\Delta(x, y)$, as discussed in Section 3. 

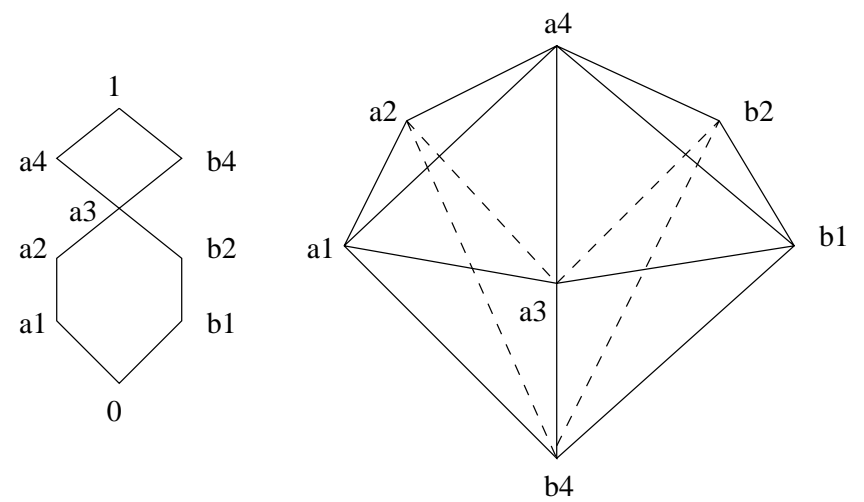

Figure 2. A poset and its order complex

Remark 1.1. We will sometimes say that a poset $P$ possesses a topological or geometric property (such as shellability), by which we mean that its order complex possesses this property.

Section 2 gives a way of constructing a discrete Morse function on the order complex of any finite poset with $\hat{0}$ and $\hat{1}$ from any lexicographic order on its saturated chains. In particular, we provide an acyclic matching on the face poset of the order complex of $P$ (not to be confused with the original poset $P$ ). To this end, let us partition the set of faces in $\Delta(P)$ by assigning each face to the earliest facet containing it, i.e. by the partitioning

$$
\Delta(P)=F_{1} \uplus\left(F_{2} \backslash F_{1}\right) \uplus \cdots \uplus\left(F_{k} \backslash\left(\cup_{j<k} F_{j}\right)\right),
$$

where $F_{1}, \ldots, F_{k}$ is a lexicographic order (as defined below) on the facets of $\Delta(P)$ or equivalently on the saturated chains of $P$. We call the above decomposition based on a lexicographic order a lexicographic decomposition of the order complex. Our approach will be to give a matching on the set of faces in each piece $F_{j} \backslash\left(\cup_{i<j} F_{i}\right)$ of the decomposition such that (1) the union of these matchings is an acyclic matching for all of $\Delta(P)$ and (2) each $F_{j} \backslash\left(\cup_{i<j} F_{i}\right)$ contributes at most one critical cell. We will utilize structure on $F_{j} \backslash\left(\cup_{i<j} F_{i}\right)$ resulting from the use of a lexicographic order on facets in much the same way that lexicographic shellability works, but without requiring our posets to be shellable. (See Section 4 for the connection between discrete Morse functions and shellings.) In 3], Billera and Hetyei made use of this same structure resulting from a poset lexicographic order in their characterization of the bounding hyperplanes for the cone of flag vectors for the set of all graded posets.

Definition 1.2. A poset lexicographic order is a total ordering of facets with the following property: let two facets $F_{1}, F_{2}$ share a face $\sigma$ of ranks $1,2, \ldots, i$, and let $\tau \subseteq F_{1}$ and $\mu \subseteq F_{2}$ be faces of ranks $1,2, \ldots, i+1$ such that $\tau \neq \mu$. If $F_{1}$ precedes $F_{2}$, then any facet containing $\tau$ must come before any facet containing $\mu$.

Lexicographic orders may be obtained by labeling Hasse diagram edges with positive integers and then lexicographically ordering the sequences of labels which are associated to the saturated chains. Such a labeling is called an edge-labeling, and when this gives rise to a certain type of shelling (see Section 4) which was 
introduced in 4], the labeling is called an EL-labeling. Recall that the Hasse diagram of $P$ is the graph whose vertices are elements of $P$ and whose edges are pairs $(u, w)$ of comparable poset elements with no intermediate comparable elements, i.e. pairs satisfying $u<w$ such that there is no intermediate $v$ satisfying $u<v<$ $w$. We denote such a minimal comparability by $u \prec v$ and call this a covering relation. Many important classes of posets possess EL-labelings (e.g. see 22 for supersolvable lattices, 4 for geometric lattices (a class of posets which includes all intersection lattices of central hyperplane arrangements, and so gives information about the cohomology of the complement of such an arrangement), or see Björner's survey article [6] for additional references).

It is easy to show that any lexicographic order may be achieved by a slight generalization of edge-labeling, known as chain-labeling. A chain-labeling is a labeling of Hasse diagram edges in which the label assigned to an edge $u \prec v$ is allowed to depend on the choice of rooted chain $\hat{0} \prec u_{1} \prec \cdots u_{k}=u$ leading up to the covering relation $u \prec v$. Chain-labeling was introduced in [7, [8] as part of a tool called CL-shellability, which generalized EL-shellability and enabled Björner and Wachs to prove that the Bruhat order of any Coxeter group is shellable (and in particular that each interval is homeomorphic to a sphere). For other interesting examples of CL-shellable posets, see [25] for the poset of direct sum decompositions of a finite-dimensional vector space over $F_{q}$, or [21] for the subgroup lattice of a finite, solvable group. From our viewpoint, EL-labelings and CL-labelings will give rise to particularly well-behaved lexicographic discrete Morse functions: it is well known that such a labeling for a poset $P$ implies that each interval in $P$ is collapsible or homotopy equivalent to a wedge of spheres of top dimension, with these spheres indexed by the saturated chains whose label sequences are strictly decreasing in value at each step.

Remark 1.2. For convenience, we will also require that any two covering relations $u \prec v$ and $u \prec w$ with distinct elements $v \neq w$ covering the same element $u$ (in the case of an edge-labeling) or covering the same root $\hat{0} \prec u_{0} \prec \cdots \prec u_{k}=u$ (in the case of a chain-labeling) be assigned distinct labels, so that the edge-labeling or chain-labeling gives a total order on saturated chains.

Translating from acyclic matchings to real-valued functions, a lexicographic discrete Morse function will assign smaller values to faces belonging to lexicographically earlier facets. Each time a facet is inserted in lexicographic order, at most one of the new faces it adds to the complex is critical, so the Betti numbers cannot change very much each time a facet is inserted. If the homotopy type changes, then either a new cycle is created at some dimension, or an old cycle becomes a boundary. This reflects the fact that each facet overlaps with the union of earlier ones in either a sphere or a disk, by virtue of our use of a lexicographic order on facets (cf. 3] or Proposition 2.1).

In Section 5, we use a chain-labeling to construct a lexicographic order on the poset $\Pi_{n} / S_{\lambda}$ of partitions of a multiset. Critical cells in the resulting lexicographic discrete Morse function live in various dimensions, and the sequence of critical cell dimensions, listed in the order that they are inserted in the complex, is not a nonincreasing sequence. Thus, we cannot conclude directly from our original Morse function that $\Pi_{n} / S_{\lambda}$ has the homotopy type of a wedge of spheres. However, for certain classes of $\lambda$ we are able to cancel all the lower-dimensional critical cells in order to show that the order complex is homotopy equivalent to a wedge of 
spheres of top dimension. We cancel critical cells in pairs $\tau^{(p+1)}, \sigma^{(p)}$ by proving there is a unique gradient path from $\tau$ to $\sigma$. The critical cells which we pair together are chosen to agree up to a high rank, forcing any gradient path from $\tau$ to $\sigma$ to preserve all the lower ranks where the cells agree, aiding us in proving uniqueness. Our main tool for proving uniqueness is then the use of a permutation statistic based on inversions in the label sequences assigned to the saturated chains; each step in a gradient path eliminates a single inversion allowing us to associate reduced expressions for permutations to gradient paths and then to use the fact that a cyclic shift permutation has a unique reduced expression and that its indices are decreasing to conclude that an associated gradient path is unique. We hope that our approach to this example may also be useful for other posets, particularly when they have the homotopy type of a wedge of spheres concentrated in a single dimension, whether or not they are Cohen-Macaulay.

Ziegler previously showed that the poset of partitions of an integer (a special case of our multiset partition poset) is not Cohen-Macaulay and hence not shellable 26]; lexicographic discrete Morse functions relax the requirements of shellability, enabling us to prove that $\Delta\left(\Pi_{n} / S_{\lambda}\right)$ is homotopy equivalent to a wedge of spheres of top dimension when $\lambda$ is of hook-shape (see Section 5 for definitions).

Question 1.1. Is the poset of partitions of a multiset always homotopy equivalent to a wedge of spheres of top dimension? Does our proof for $\lambda$ of hook-shape extend to all $\lambda$, i.e. is it true that the puzzle in Section 5.8 has no solutions?

Examples of lexicographic discrete Morse functions for several other posets appear in [15] and 17]. All of these examples use very natural edge-labelings, and they lead us to believe that the most natural of labelings will often yield Morse functions which may be transformed by gradient path reversal into perfect Morse functions, i.e. ones in which the Morse numbers equal the Betti numbers. Thus, the original complex may be collapsed onto a cell complex which computes its homotopy type, in that each cell actually contributes to one of the Betti numbers.

\section{LeXICOGRAPHIC DISCRETE MORSE FUnCTIONS}

Given a lexicographic order $F_{1}, \ldots, F_{k}$ on the facets in the order complex of a graded poset with $\hat{0}$ and $\hat{1}$, we specify a resulting matching on the faces in $F_{j} \backslash$ $\left(\cup_{i<j} F_{i}\right)$ for $1 \leq j \leq k$ such that the union of these matchings will be an acyclic matching on $\Delta(P)$. Each facet contributes at most one critical cell; it contributes one when the facet insertion changes the homotopy type and does not contribute any when $\cup_{i \leq j} F_{i}$ may be collapsed onto $\cup_{i<j} F_{i}$.

This matching will require some notation. If $F_{i}$ precedes $F_{j}$ in the lexicographic order on facets, denote this by $F_{i}<_{l e x} F_{j}$. Denote the lexicographically earliest facet containing a face $g$ by $e g$, since $e g$ may be thought of as an extension of a poset chain $g$ to a saturated chain $e g$. If $e g<_{l e x} e h$, then we also say $g<_{l e x} h$, and it will turn out that $f(g)<f(h)$ for every discrete Morse function $f$ which is consistent with the acyclic matching we are about to construct. If a face $a$ is matched with a face $b$, denote this by $a \sim b$. We will specify the matching by describing it for the fibres $e^{-1}\left(F_{j}\right)$ for each saturated chain $F_{j}$.

Remark 2.1. Given a saturated chain $F_{j}$ and a lexicographic order $F_{1}, \ldots, F_{k}$ on the facets, each maximal face in $F_{j} \cap\left(\cup_{i<j} F_{i}\right)$ consists of a subchain of $F_{j}$ given by skipping a single interval of consecutive ranks in $F_{j}$. 
We refer to these intervals in ranks as the minimal skipped intervals of $F_{j}$, and we denote this set of intervals by $I\left(F_{j}\right)$. We call the number of ranks which are skipped to obtain a maximal face in $F_{j} \cap\left(\cup_{i<j} F_{i}\right)$ the height of the interval. A minimal skipped interval is called nontrivial if it skips more than one rank.

Remark 2.2. One way to determine the minimal skipped intervals for a particular $F_{j}$ is by considering each covering relation $u \prec v$ in $F_{j}$ in turn; if there is some $u \prec v^{\prime}$ which comes lexicographically earlier, then one obtains a face in $F_{j} \cap\left(\cup_{i<j} F_{i}\right)$ by taking the intersection of $F_{j}$ with any facet $F_{i}$ which includes $u \prec v^{\prime}$, agrees with $F_{j}$ below $u$, and agrees with $F_{j}$ above $w \in F_{j}$ for some $w>v^{\prime}$ of smallest possible rank in $F_{j}$. All maximal faces in $F_{j} \cap\left(\cup_{i<j} F_{i}\right)$ arise in this way. Natural labelings often seem to allow simple classification of minimal skipped intervals.

In [3], the collection $I\left(F_{j}\right)$ of minimal skipped intervals for $F_{j}$ is called the interval system of $F_{j}$, and it is used to determine the bounding hyperplanes for the cone of all possible flag $f$-vectors for graded posets. We shall also use interval systems, in our case to describe the faces in $F_{j} \backslash\left(\cup_{i<j} F_{i}\right)$, so as to construct an acyclic matching on the set of faces in each $F_{j} \backslash\left(\cup_{i<j} F_{i}\right)$. Thus, our lexicographic discrete Morse function construction will also work for balanced simplicial complexes which have the above property of poset lexicographic orders that every maximal face in $F_{j} \cap\left(\cup_{i<j} F_{i}\right)$ skips a single interval. See [14 for a notion of lexicographic order on balanced simplicial complexes. Not all lexicographic orders on simplicial complexes have such an interval system structure.

Note that a face in $F_{j}$ also belongs to a lexicographically earlier facet if it entirely misses any of the minimal skipped intervals of $F_{j}$, so the faces in $F_{j} \backslash\left(\cup_{i<j} F_{i}\right)$ are those faces within $F_{j}$ which hit every minimal skipped interval of $F_{j}$. Our matching will be specified in terms of how a closely related set of nonoverlapping intervals called $J\left(F_{j}\right)$ are hit. Figure 3 provides an example in which $I\left(F_{j}\right)=J\left(F_{j}\right)$. The skipped intervals in $I\left(F_{j}\right)$ for the darkened saturated chain $F_{j}$ have rank sets $\{1\},\{2,3\},\{4,5\},\{6\}$ and the faces in $F_{j} \backslash\left(\cup_{i<j} F_{i}\right)$ are depicted to the right of the poset by showing for each face which ranks it hits in each of the intervals in $I\left(F_{j}\right)$. The critical cell is listed first, followed by pairs of critical cells which are to be matched.

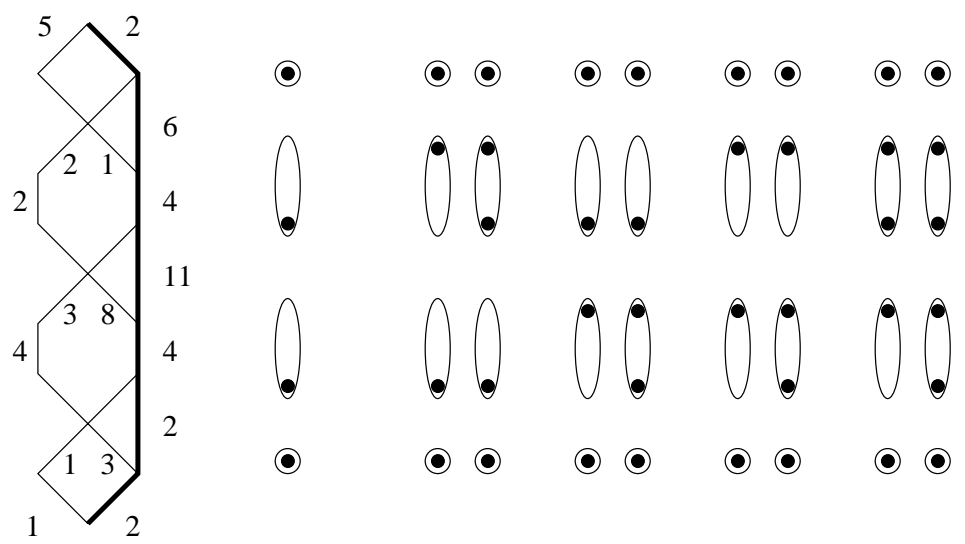

Figure 3. A matching example 
Now let us informally describe the matching. If some vertex $v \in F_{j}$ does not belong to any minimal skipped interval of $F_{j}$, then including or excluding $v$ has no bearing on whether a face of $F_{j}$ also belongs to a lexicographically earlier facet, so we choose the lowest rank such that $v \in F_{j}$ and match faces including $v$ with those excluding it. In this case, the matching on $F_{j} \cap\left(\cup_{i<j} F_{i}\right)$ has no critical cells. Otherwise, every vertex in $F_{j}$ belongs to some minimal skipped interval, and then we truncate the intervals in $I\left(F_{j}\right)$ to obtain a collection $J\left(F_{j}\right)$ of nonoverlapping intervals as follows: we include in $J\left(F_{j}\right)$ the lowest rank interval from $I\left(F_{j}\right)$ in its entirety, truncate all the remaining intervals to avoid overlap with this interval, discard any intervals which are no longer minimal and then iterate the procedure on the truncated intervals which are still minimal among the set of skipped intervals (i.e. which do not contain any of the other intervals) to obtain a collection $J$ of nonoverlapping intervals.

The unique critical cell in the $F_{j}$-piece of the lexicographic decomposition will consist of the minimal elements of all the intervals in $J\left(F_{j}\right)$. The other cells in $F_{j} \backslash\left(\cup_{i<j} F_{i}\right)$ are matched based on the first interval in $J\left(F_{j}\right)$ where they deviate from the critical cell. This deviation comes either by leaving out the minimal element of the interval or else by including other vertices along with the minimal element; we match a face which omits the minimal element in the interval with a face which has just that vertex added. Notice that the faces in $F_{j} \backslash\left(\cup_{i<j} F_{i}\right)$ need not hit all the $J$-intervals, but our matching relies on the fact that each such face does necessarily include an element from the first $J$-interval where it deviates from the critical cell. To see this, notice that this first $J$-interval is contained in some $I$-interval which may extend downward below the $J$-interval, and that the face must hit this $I$-interval; the face cannot hit the portion of the $I$-interval which is truncated to obtain the $J$-interval, because we assume that the face agrees with the critical cell below the $J$-interval in question.

It may happen that the intervals in $I(F)$ cover the saturated chain even though the intervals in $J(F)$ do not cover it, in which case our construction does produce a critical cell. As an example, consider a poset of rank 5 and a facet with minimal skipped intervals $[1,2],[2,3],[3,4]$ in $I(F)$, which in turn yields truncated intervals $[1,2]$, [3] for $J(F)$ since [3,4] strictly contains the interval [3] and thus is discarded. Note that the intervals in $J(F)$ do not cover the vertex of rank 4, but nonetheless the facet contributes a critical cell of ranks 1,3 , namely the minimal ranks of the intervals in $J(F)$, because $I(F)$ did cover the facet.

Let us formalize the matching construction. For each saturated chain $F_{k}$, we derive from the set $I\left(F_{k}\right)$ of minimal skipped intervals a collection $J\left(F_{k}\right)$ of disjoint intervals by the following algorithm (which is initialized to $J=\emptyset$ ):

(1) Add to $J$ the interval $(u, v)$ in $I$ with $u$ of smallest possible rank.

(2) Replace $I$ by the restriction of $I$ to ranks above $r k(v)$.

(3) Delete from $I$ those skipped intervals which are no longer minimal.

(4) Repeat these steps until $I=\emptyset$.

This algorithm outputs the collection $J$ of disjoint intervals. If $I$ does not include all the vertices of $F_{k}$, let $j_{0}$ be the set of uncovered elements (in which case the facet $F_{k}$ has contractible overlap with $\cup_{i<k} F_{i}$ because the uncovered vertices of $F_{k}$ are cone points in this overlap as well as in $\left.F_{k} \backslash\left(\cup_{i<k} F_{i}\right)\right)$.

If $j_{0} \neq \emptyset$, then let $\rho_{0}$ denote the lowest rank element whose rank is in $j_{0}$, and match $h \sim h \backslash\left\{\rho_{0}\right\}$ for each face $h \in e^{-1}\left(F_{k}\right)$ containing the element $\rho_{0}$. Otherwise, 
let $\rho_{i}$ denote the lowest rank element from interval $j_{i} \in J$ for each $i>0$. Let $[r]$ be the set of indices on the intervals in $J$. Now define a map

$$
\tau: e^{-1}\left(F_{k}\right) \rightarrow[r] \cup\{\infty\}
$$

from chains $h$ that extend to $F_{k}$ to indices of $J$-intervals by

$$
h \mapsto \min _{i}\left\{h \cap j_{i} \neq\left\{\rho_{i}\right\}\right\},
$$

letting $\tau h=\infty$ when the set $\left\{h \cap j_{i} \neq\left\{\rho_{i}\right\}\right\}$ is empty. The matching is defined by $h \sim h \backslash\left\{\rho_{\tau h}\right\}$ for $\rho_{\tau h} \in h$ and $h \sim h \cup\left\{\rho_{\tau h}\right\}$ otherwise. This leaves $h$ unmatched when $\tau h=\infty$, namely when $j_{0}$ is empty and $h$ is comprised of exactly the minimal elements $\rho_{i}$ of each interval.

Theorem 2.1. The above construction gives an acyclic matching on $\Delta(P)$.

Proof. Let us begin with the case where the $I$-intervals and $J$-intervals agree, namely where the $I$-intervals are already nonoverlapping. Each face is matched with at most one other face by the bijection between what we will call "hollow" cells and "doubly-hit" cells within any fibre $e^{-1}\left(F_{k}\right)$. A cell is hollow if it lacks the minimal rank vertex in the first interval where it differs from the critical cell; a cell is doubly-hit if instead it includes the minimal rank vertex and at least one other vertex in this interval. Our observation above about every face in $F_{k} \backslash\left(\cup_{i<k} F_{i}\right)$ hitting the first $J$-interval where it differs from the critical cell guarantees that this indeed gives a matching on faces in $F_{k} \backslash\left(\cup_{i<k} F_{i}\right)$.

To check that our matching is acyclic, we verify (1) that it is acyclic on each $F_{k} \backslash\left(\cup_{i<k} F_{i}\right)$ and $(2)$ that there are no directed cycles involving multiple pieces of our decomposition, i.e. multiple fibres. Fact (2) follows from the observation that every edge between distinct fibres is oriented from the later fibre to the earlier one. To verify (1), suppose there is a cycle in a single fibre, and consider any upward oriented edge $a \prec b$ in the cycle. There must then be a downward edge from $b$ to some other $c$. A cycle may only involve two ranks of the face poset since two consecutive upward edges are impossible in a matching. Thus, $a$ and $c$ must be hollow while $b$ must be doubly-hit. Furthermore, $c$ is obtained from $b$ by deleting some rank above the rank where $a$ and $b$ differ or else $c$ would belong to an earlier orbit. This choice of deletion precludes $c$ being hollow unless we obtain $c$ by deleting from $b$ one element on the interval where $a$ and $b$ first differ, and $c$ is left with only the minimal element on that interval. Continuing in this fashion, our cycle must produce cells that agree with the critical cell (if there is one) to higher and higher ranks, and thus the cycle may never be completed. The point is that we may never move downward in the face poset by deleting the unique element covering an interval and also may never move upward by adding to an interval where we only have the minimal element, so such intervals may never be altered.

Now suppose that the $J$-intervals differ from the $I$-intervals. We claim that the above matching on faces hitting all the $J$-intervals extends to all of $e^{-1}\left(F_{k}\right)$. What requires special consideration are faces in $F_{k} \backslash\left(\cup_{i<k} F_{i}\right)$ that do not hit all of the $J$-intervals, despite hitting all the $I$-intervals. Note that any rank $r$ which gets truncated from an $I$-interval when the interval is converted to a $J$-interval $j$ also must belong to another $I$-interval at lower ranks, and this lower $I$-interval must be one that also gives rise to a $J$-interval, which we denote $j^{\prime}$. However, such an $r$ cannot be the lowest rank in this new $I$-interval. Thus, a face which includes rank $r$ will differ from the critical cell on the resulting $J$-interval and hence be matched 
by our construction, unless rank $r$ is the lowest rank in the resulting $J$-interval. However, this contradicts $j$ being a minimal skipped interval at all, since it would strictly contain $j^{\prime}$.

Each fibre $e^{-1}\left(F_{k}\right)$ will have at most one critical cell, as described next.

Theorem 2.2. Each facet contributes at most one critical cell. The set of faces in $F_{k} \backslash\left(\cup_{i<k} F_{i}\right)$ includes a critical cell exactly when the attachment of $F_{k}$ changes the homotopy type of the complex, i.e. when every vertex in $F_{k}$ belongs to at least one of the intervals in $I\left(F_{k}\right)$.

Proof. If some node is not in any minimal skipped interval of $F_{k}$, then the minimal rank such node $v$ is a cone point in $F_{k} \cap\left(\cup_{i<k} F_{i}\right)$ and is used to match faces including $v$ with those excluding $v$, so $F_{k} \backslash\left(\cup_{i<k} F_{i}\right)$ has no critical cells. In this case, the complex $F_{1} \cup \cdots \cup F_{k}$ of faces belonging to one of the first $k$ facets collapses onto $F_{1} \cup \cdots \cup F_{k-1}$, so the homotopy type is unchanged. If every node in $F_{k}$ belongs to at least one of its minimal skipped intervals, then our acyclic matching has a single critical cell comprised of the minimal ranks of the $J$-intervals. Thus, exactly one Morse number changes, implying that the reduced Euler characteristic changes and hence the homotopy type also must change. Alternatively, one may apply the next proposition.

Corollary 2.1. When $F_{j}$ contributes a critical cell, then the dimension of the critical cell is one less than the number of J-intervals for $F_{j}$.

The following proposition follows from a similar argument to our proof of Theorem 2.1 (though this was not the approach of Billera and Hetyei). That is, one may give an acyclic matching on each $F_{j} \cap\left(\cup_{i<j} F_{i}\right)$ such that $(1) F_{j} \cap\left(\cup_{i<j} F_{i}\right)$ has no critical cells when $F_{j} \backslash\left(\cup_{i<j} F_{i}\right)$ has no critical cells, and (2) otherwise, the critical cells in $F_{j} \cap\left(\cup_{i<j} F_{i}\right)$ are the proper faces of the unique critical cell in $F_{j} \backslash\left(\cup_{i<j} F_{i}\right)$.

Proposition 2.1 (Billera-Hetyei). Given a lexicographic order $F_{1}, \ldots, F_{k}$, the intersection $F_{j} \cap\left(\cup_{i<j} F_{i}\right)$ is either homotopy equivalent to a sphere or is collapsible.

From this, a simple Mayer-Vietorus argument yields:

Corollary 2.2. Each facet attachment changes at most one Betti number, and changes its value by at most one.

We conclude this section with a useful fact for proving the uniqueness of a gradient path from a critical cell $\tau^{(p+1)}$ to a critical cell $\sigma^{(p)}$. First, it is helpful to note that any gradient path from $\tau^{(p+1)}$ to $\sigma^{(p)}$ must alternate between only the ranks $p+1$ and $p$ (cf. 13) since it cannot have two consecutive upward steps (since the upward edges comprise a matching) and also cannot end with an upward step (since $\sigma^{(p)}$ is not in the matching, so there are no upward edges leading to $\sigma^{(p)}$ ).

Proposition 2.2. If critical cells $\sigma^{(p)}$ and $\tau^{(p+1)}$ agree up through rank $r$, then every gradient path from $\tau$ to $\sigma$ leaves ranks $1,2, \ldots, r$ unchanged.

Proof. If a rank $r^{\prime}$ below $r+1$ is deleted in a gradient path step $\tau_{i} \rightarrow \sigma_{i}$, then $e \sigma_{i}<_{l e x} e \sigma$. This is because the first gradient path step deleting an element at any rank $r^{\prime} \leq r$ will be immediately followed by an upward step inserting an element at rank $r^{\prime}$ or lower that belongs to a strictly earlier saturated chain. To see this, recall that the element at rank $r^{\prime}$ was the lowest element of $e \tau$ in the interior of one of its 
$J$-intervals, and that none of the earlier upward (matching) steps will ever insert another element on this $J$-interval. Thus, the chain obtained by deleting rank $r^{\prime}$ belongs to a saturated chain which is strictly earlier in lexicographic order than both $e \tau$ and $e \sigma$. This makes it impossible for our Morse function to decrease at every remaining step on the gradient path to $\sigma$, a contradiction.

In Section 5, we shall establish a notion of rank inversion for the poset of partitions of a multiset in terms of labels on its covering relations.

\section{Möbius Functions}

Let us observe, via the Morse inequalities and our description of critical cells in terms of skipped intervals in saturated chains, that the Möbius function $\mu(\hat{0}, \hat{1})$ may be computed by the following formula:

\section{Proposition 3.1.}

$$
\mu_{P}(\hat{0}, \hat{1})=\sum_{\sigma \in \operatorname{Crit}(\Delta)}(-1)^{|J(e \sigma)|+1},
$$

where the sum is over critical cells $\sigma$ of a lexicographic discrete Morse function, letting $J(C)$ denote the collection of disjoint skipped intervals in a saturated chain $C$ obtained from the original skipped intervals $I(C)$.

Proof. Consider the alternating sum $\sum_{i=-1}^{\operatorname{dim}(\Delta)}(-1)^{i} m_{i}$ of criticals cells of various dimensions in a reduced discrete Morse function, i.e. one which includes the empty set. Now recall from the Morse inequalities that

$$
\sum_{i=-1}^{\operatorname{dim}(\Delta)}(-1)^{i} m_{i}=\sum_{i=-1}^{\operatorname{dim}(\Delta)}(-1)^{i} b_{i}=\tilde{\chi}(\Delta) .
$$

Each saturated chain $m$ has at most one critical cell $\sigma$, and this satisfies $\operatorname{dim}(\sigma)=$ $|J(m)|-1$.

The facets $m$ contributing critical cells are exactly those where every vertex in $m$ belongs to some interval in $I(m)$. Thus, one might compute $\mu_{P}(\hat{0}, \hat{1})$ by characterizing facets which have a critical cell and determining dimension parities. This may be an easy way (for instance) to show the Möbius function is 0 for certain classes of poset intervals; we give one example of such a computation for the poset of partitions of a multiset later.

Remark 3.1. To obtain $\mu_{P}(u, v)$, simply restrict the lexicographic order to the interval $(u, v)$ and consider the resulting lexicographic discrete Morse function there.

One could also derive a lexicographic order on any rank-selected subposet $P^{S}$, which in turn would give a lexicographic discrete Morse function on $P^{S}$, giving an expression for the rank-selected Möbius function. We have not looked into whether there is any useful relationship between the critical cells on intervals or rank-selected subposets and the critical cells for the entire poset.

\section{Relation to Shellability}

This section makes explicit how to construct a discrete Morse function from an arbitrary shelling; lexicographic discrete Morse functions generalize the notion of a lexicographic shelling in exactly the same fashion. 
Definition 4.1. A simplicial complex $\Delta$ is pure of dimension $d$ if all its maximal faces are $d$-dimensional. A shelling is a facet ordering $F_{1}, \ldots, F_{k}$ on $\Delta$ such that $F_{j} \cap\left(\cup_{i<j} F_{i}\right)$ is pure of codimension one for $2 \leq j \leq k$.

A shelling gives a way of building up a pure complex $\Delta$ by sequentially inserting facets such that each facet either attaches along its entire boundary or leaves the homotopy type unchanged, implying that $\Delta$ is collapsible or homotopy equivalent to a wedge of spheres of top dimension (and has a similar local property called Cohen-Macaulayness). The following is very similar to Theorem 4.2 in [11], but is included for the sake of completeness.

Proposition 4.1. If $F_{1}, \ldots, F_{k}$ is a shelling order for the facets in a pure simplicial complex $\Delta$ of dimension d, then $\Delta$ has a discrete Morse function whose critical cells are the facets $F_{j}$ of $\Delta$ such that $F_{j} \cap\left(\cup_{i<j} F_{i}\right)$ is the entire boundary of $F_{j}$.

Proof. Since $F_{1}, \ldots, F_{k}$ is a shelling, $F_{j} \cap\left(\cup_{i<j} F_{i}\right)$ is pure of codimension one, so its maximal faces each omit a single vertex of $F_{j}$. Hence, the intersection is either the entire boundary of $F_{j}$ or it has at least one cone point, namely a vertex which is not omitted by any of the maximal faces in $F_{j} \cap\left(\cup_{i<j} F_{i}\right)$. In either case, we give a matching on the faces in $F_{j} \backslash\left(\cup_{i<j} F_{i}\right)$ so that the union of these matchings is an acyclic matching on $\Delta$ with the desired set of critical cells unmatched. When $F_{j} \cap\left(\cup_{i<j} F_{i}\right)$ has one or more cone points, choose any such cone point $v$. Match each face in $F_{j} \backslash\left(\cup_{i<j} F_{i}\right)$ which includes $v$ with the face in $F_{j} \backslash\left(\cup_{i<j} F_{i}\right)$ obtained by deleting $v$. Note that every face in $F_{j} \backslash\left(\cup_{i<j} F_{i}\right)$ is used in this matching exactly once. When $F_{j} \cap\left(\cup_{i<j} F_{i}\right)$ is the entire boundary of $F_{j}$, then the only face in $F_{j} \backslash\left(\cup_{i<j} F_{i}\right)$ is the facet $F_{j}$, and it is left unmatched.

It is easy to check that the union of these matchings is an acyclic matching on the face poset, since matching edges (i.e. the edges which get directed upward) are covering relations $u \prec v$ where $u, v$ are both contributed by the same facet. Hence, there are no covering relations $u \prec v$ with $v$ belonging to an earlier facet than the facet to which $u$ belongs. Hence, any directed cycle would live in some $F_{j} \backslash\left(\cup_{i<j} F_{i}\right)$. This is impossible because each of the upward oriented edges in $F_{j} \backslash\left(\cup_{i<j} F_{i}\right)$ inserts the same cone point $v$ but no downward edges may delete $v$.

Whenever a lexicographic order on facets gives rise to a shelling, we call this a lexicographic shelling. This is equivalent to Kozlov's notions of EC-shellability (for edge-labelings) and CC-shellability (for chain-labelings), as introduced in [18].

Proposition 4.2. A lexicographic order on facets is a shelling order if it gives rise to a lexicographic discrete Morse function in which all minimal skipped intervals have height one.

Proof. Specialize the above proof to this situation to get a lexicographic discrete Morse function. The reverse direction is essentially shown in [18].

Kozlov's notion amounts to requiring for each interval that all but the lexicographically earliest chain have a minimal skipped interval of height one. Such an interval of height one is called a topological descent in [16, [14 because it behaves topologically like a descent would behave in an EL-labeling or CL-labeling.

\section{ON The HOMOtopy type OF $\Delta\left(\Pi_{n} / S_{\lambda}\right)$}

This section uses a particular lexicographic discrete Morse function to show for $\lambda$ of hook shape that the poset $\Delta\left(\Pi_{n} / S_{\lambda}\right)$ of partitions of a multiset ordered by 
refinement is either collapsible or homotopy equivalent to a wedge of spheres of dimension $n-3$. It seems likely that with further work this hook shape assumption may be relaxed or perhaps even removed.

Let $\Pi_{n}$ denote the poset of set partitions of $\{1,2, \ldots, n\}$ ordered by refinement. Let $\lambda=\left(\lambda_{1}, \ldots, \lambda_{k}\right)$ be a number partition of the integer $n$ into unordered parts $\lambda_{1}, \ldots, \lambda_{k}$. By convention, we list the parts in decreasing order, so they satisfy $\lambda_{1} \geq$ $\cdots \geq \lambda_{k}>0$ and $\lambda_{1}+\cdots+\lambda_{k}=n$. Denote by $S_{\lambda}$ the Young subgroup $S_{\lambda_{1}} \times \cdots \times S_{\lambda_{k}}$ of the symmetric group $S_{n}$. Notice that $S_{\lambda}$ acts on $\{1,2, \ldots, n\}$ by letting each $S_{\lambda_{i}}$ permute the values $\left\{\lambda_{1}+\cdots+\lambda_{i-1}+1, \ldots, \lambda_{1}+\cdots+\lambda_{i-1}+\lambda_{i}\right\}$ among themselves. This naturally gives rise to an order-preserving $S_{\lambda}$-action on the set partitions of $\{1,2, \ldots, n\}$, i.e. an action such that $u<v$ implies $\sigma u<\sigma v$ for each $\sigma \in S_{\lambda}$ and $u, v$ comparable elements of $\Pi_{n}$. Thus, it makes sense to consider the quotient poset $\Pi_{n} / S_{\lambda}$, i.e. the poset of partitions of a set with repetition $\left\{1^{\lambda_{1}}, \ldots, k^{\lambda_{k}}\right\}$ (which we call a multiset). Figures 4 and 5 show the posets $\Pi_{4} / S_{2} \times S_{1} \times S_{1}$ and $P_{5} \cong \Pi_{5} / S_{5}$, respectively, along with their order complexes.
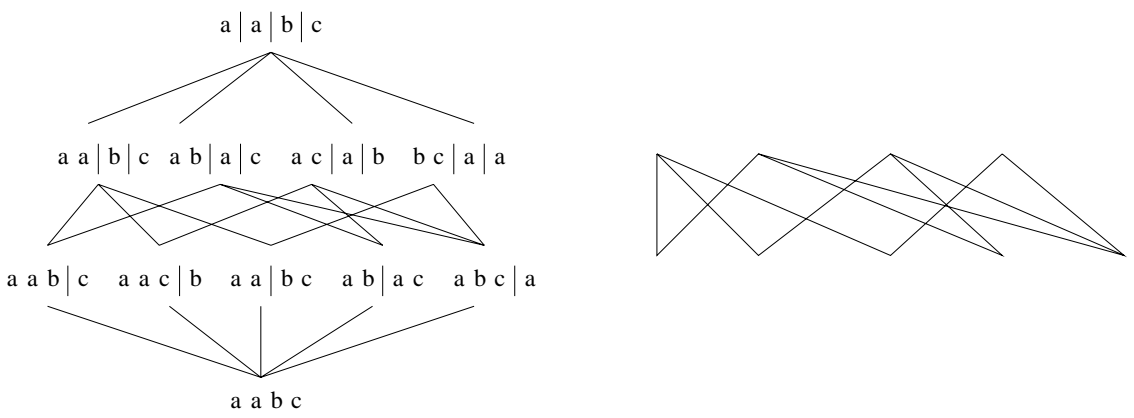

Figure $4 . \Pi_{4} / S_{2} \times S_{1} \times S_{1}$ and its (1-dimensional) order complex
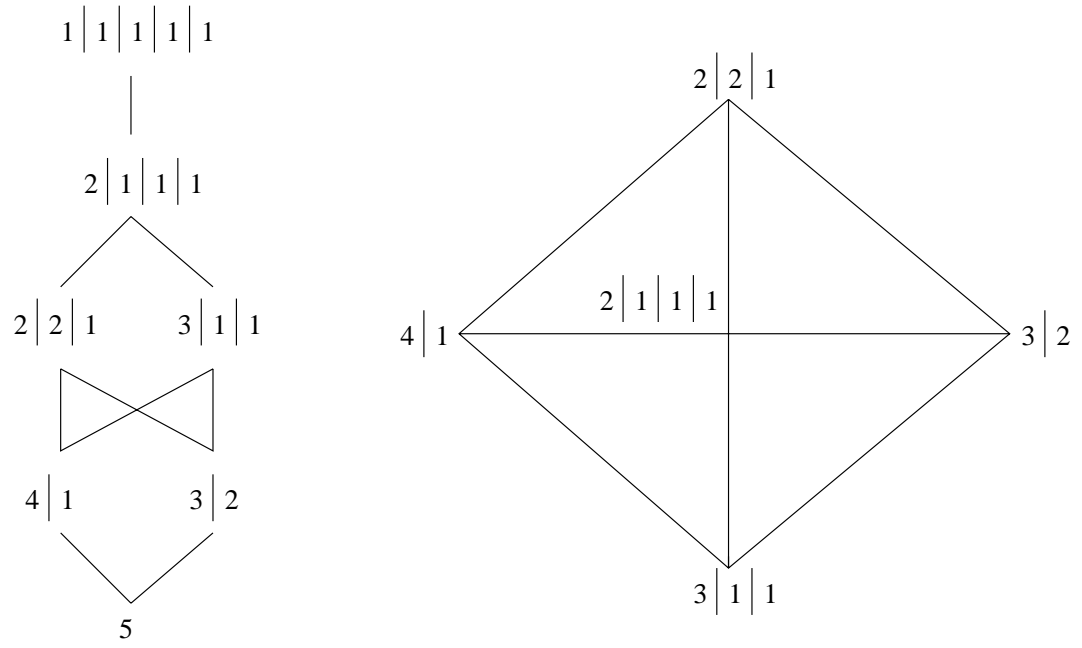

FiguRE $5 . \Pi_{5} / S_{5}$ and its (2-dimensional) order complex $\Delta\left(\Pi_{5} / S_{5}\right)$ 
We will study $\Pi_{n} / S_{\lambda}$ in the special case where $\lambda$ is a number partition of hook shape, namely one in which $\lambda_{2}=\cdots=\lambda_{k}=1$. Notice that this extrapolates between the partition lattice $\Pi_{n}$ (in which case $\lambda_{1}$ also equals 1 ) and the poset $P_{n}$ of number partitions of an integer $n$ ordered by refinement (in which case $k=1$ ).

Ziegler showed that $\Delta\left(\Pi_{n} / S_{n}\right)$ is not Cohen-Macaulay for $n \geq 19$ by exhibiting a disconnected interval of rank 3 in the poset $P_{19}$ [26, p. 218]. Recall that $\Delta$ is Cohen-Macaulay if the link of each face (including the empty face) has only top dimensional (reduced) homology; note that a disconnected graph $G$ has dimension 1 , but $\tilde{H}_{0}(G) \neq 0$, so it is not Cohen-Macaulay. At the other extreme, Gessel gave an EL-labeling for $\Pi_{n}$, implying $\Delta\left(\Pi_{n}\right)$ is homotopy equivalent to a wedge of spheres of top dimension, as discussed in [4, p. 165]. We raise the question of whether all posets $\Pi_{n} / S_{\lambda}$ are nonetheless either collapsible or homotopy equivalent to a wedge of spheres of top dimension, and give an affirmative answer for $\lambda$ of hook shape.

Example 5.1 (Ziegler). The open interval $(6|5| 3|2| 2|1,8| 7 \mid 4)$ prevents $P_{19}$ from being Cohen-Macaulay. Simply note that $8|7| 4$ may be refined to $6|5| 3|2| 1 \mid 1$ in two different ways:

$$
\begin{aligned}
& 8=6+2=5+3, \\
& 7=5+2=6+1, \\
& 4=3+1=2+2 .
\end{aligned}
$$

Any saturated chain from $\hat{0}$ to $6|5| 3|2| 2 \mid 1$ together with any saturated chain from $8|7| 4$ to $\hat{1}$ gives a face $F \in \Delta\left(P_{19}\right)$ such that $l k(F)$ is a disconnected graph, and hence has lower homology.

The remainder of this paper will be concerned with proving the following theorem and deducing a consequence about Möbius functions.

Theorem 5.1. If $\lambda$ is a partition of hook-shape, then the poset $\Pi_{n} / S_{\lambda}$ of partitions of the multiset $\left\{1^{\lambda_{1}}, \ldots, k^{\lambda_{k}}\right\}$ ordered by refinement is either collapsible or has the homotopy type of a wedge of spheres concentrated in top dimension.

Here is an outline of the proof:

(1) Give a lexicographic order on saturated chains based on a chain-labeling. We shall then consider the resulting lexicographic discrete Morse function.

(2) Classify lower critical cells (i.e. critical cells not of top dimension) by classifying the three ways in which nontrivial minimal skipped intervals may arise in posets $\Pi_{n} / S_{\lambda}$. This will enable us to pair lower critical cells based on the structure of their highest rank nontrivial minimal skipped interval. One of these classes of nontrivial minimal skipped interval cannot occur in critical cells for $\lambda$ of hook shape.

(3) For the two types of lower critical cells $\sigma$ that occur in hook shapes, we prove (by explicit construction) the existence of a partner critical cell $\tau$ such that either there is a gradient path from $\sigma$ to $\tau$ (for $\operatorname{dim}(\sigma)=\operatorname{dim}(\tau)+1$ ) or from $\tau$ to $\sigma$ (for $\operatorname{dim}(\tau)=\operatorname{dim}(\sigma)+1$ ). For the remaining class of lower critical cells, we still construct partner cells, and it is open whether or not these partner cells are always critical.

(4) Prove that for each pair $\sigma, \tau$ of partner (critical) cells there is a unique gradient path from $\tau$ to $\sigma$. This implies that $\sigma$ and $\tau$ may indeed cancel 
by reversing this gradient path to obtain a larger acyclic matching. This uniqueness of gradient paths follows from our use of a statistic called the inversion set of a cell which decreases at every step in a gradient path.

(5) Choose how to pair critical cells (in a way related to step 4) so that no two critical cells have the same partner. Furthermore, we show that partner critical cells occur consecutively among the set of all critical cells in lexicographic order, so cancellation proceeds as desired.

The third step in the proof uses the assumption that $\lambda$ is of hook-shape, so at the end of that section we indicate what exactly would be needed to generalize the proof to all $\lambda$. It seems likely that the hook-shape assumption can at least be weakened. Now let us turn to the details of the proof.

5.1. A lexicographic order on saturated chains. We will use a chain-labeling to order the saturated chains, so each covering relation $v_{i-1} \prec v_{i}$ is given a label which may depend on the rooted chain $\hat{0} \prec v_{1} \prec \cdots \prec v_{i-1}$ which it extends to $\hat{0} \prec \cdots \prec v_{i-1} \prec v_{i}$; saturated chains are ordered by the lexicographic order on their label sequences $\lambda_{1} \lambda_{2} \cdots \lambda_{n}$, where $\lambda_{i}$ is the label for $v_{i-1} \prec v_{i}$. In particular, the label we assign to $u \prec v$ in $\Pi_{n} / S_{\lambda}$ will depend on an ordering of the blocks of $u$ which is determined inductively by the choice of root for $u$. That is, we obtain a block order for $v_{i}$ from a block order for $v_{i-1}$ whenever $v_{i-1} \prec v_{i}$ by preserving the order in $v_{i-1}$ in all but the one block which gets refined to obtain $v_{i}$ and replacing this block by the two resulting blocks as follows: if there is more than one block in $v_{i-1}$ of the type to be refined, then we refine the leftmost such block, and when replacing it by the two new blocks we place the smaller one to the left of the larger one. We determine which of these two blocks is smaller by using the length-lex block order (defined in [16]), namely by letting $B_{1}<B_{2}$ for $\left|B_{1}\right|<\left|B_{2}\right|$ or for $\left|B_{1}\right|=\left|B_{2}\right|$ and $w_{B_{1}}<w_{B_{2}}$ in the lexicographic order on words where $w_{B}$ is the word obtained from the multiset $B$ by arranging the letters in increasing order.

When a covering relation $u \prec v$ splits a block $B$ into children $B_{1}, B_{2}$ such that $B_{1}<B_{2}$ in length-lex order, let us denote $B_{1}$ by $L_{u \prec v}$ and $B_{2}$ by $R_{u \prec v}$, since $B_{1}$ is the left child and $B_{2}$ is the right child. The rooted covering relation $v_{i-1} \prec v_{i}$ is then labeled by the ordered pair $\left(n_{i}, L_{v_{i-1} \prec v_{i}}\right)$ consisting of the position $n_{i}$ of the newly inserted bar together with the block word immediately to the left of the new bar in $v_{i}$; the bar position $n_{i}$ has higher precedence in the label. As an example of our labeling, the saturated chain

$$
a a b b b<a b|a b b<a b| b|a b<a| b|b| a b<a|b| b|a| b
$$

in $\Pi_{5} / S_{(3,2)}$ is labeled $((2, a b),(3, b),(1, a),(4, a))$. We will often use a more compact notation for saturated chains, namely we will write the most refined element of the chain with bars separating the elements labeled by the rank at which the bars are inserted in the course of successively refining the partition. For instance, the above chain could be more succinctly expressed as $\left.\left.\left.\left.a\right|_{3} b\right|_{1} b\right|_{2} a\right|_{4} b$.

5.2. Classification of lower critical cells in terms of skipped intervals. When a critical cell is not a facet, it must have at least one nontrivial minimal skipped interval (i.e. one that skips multiple ranks). In this section, we classify the critical cells which are of smaller than top possible dimension according to the structure of their nontrivial minimal skipped interval appearing at the highest rank. We will refer to critical cells which are not top-dimensional as lower critical cells. 
There are three fundamental ways in which nontrivial minimal skipped intervals may arise (as well as hybrid mixtures of these). An instance of type three is discussed in Example 5.2 in later sections. In each case, we have a saturated chain $u=u_{1} \prec u_{2} \prec \cdots \prec u_{k}=v$ on an interval $(u, v)$ which is not lexicographically smallest on $(u, v)$ but where $u_{i} \prec u_{i+1} \prec u_{i+1}$ is lexicographically smallest on the interval $\left(u_{i}, u_{i+2}\right)$ for each $1 \leq i<k-2$. It will be useful for us to begin by describing the two ways that extensions $u_{i} \prec u_{i+1} \prec u_{i+1}$ of the interval $\left(u_{i}, u_{i+2}\right)$ might not be lexicographically smallest on the interval $\left(u_{i}, u_{i+2}\right)$.

(1) When bars are inserted sequentially from right to left in consecutive steps $u_{i} \prec u_{i+1}$ and $u_{i+1} \prec u_{i+2}$.

(2) When a single block of $u_{i}$ is split into three pieces in $u_{i+2}$ such that bars are inserted from left to right in the chain $u_{i} \prec u_{i+1} \prec u_{i+2}$ with the resulting blocks decreasing in size from left to right, i.e. with $L_{u_{i} \prec u_{i+1}}>L_{u_{i+1} \prec u_{i+2}}$.

Following [16], we say there is a topological descent at $u_{i+1}$ in these situations where $u_{i} \prec u_{i+1} \prec u_{i+2}$ is not lexicographically smallest on $\left(u_{i}, u_{i+2}\right)$. As examples, the chain $\left.\left.a\right|_{2} a\right|_{1} a a$ has a topological descent of type 1 at rank 1 while the chain $\left.\left.b\right|_{1} a\right|_{2} c$ has a topological descent of type 2 at rank 1 . Since every rank in $e \sigma$ must belong to some interval in $I(e \sigma)$ for $\sigma$ to be critical, there must be a topological descent at every rank above the highest rank nontrivial interval in $I$.

Let us now list the three basic forms which nontrivial minimal skipped intervals $(u, v)$ may take. To get such an interval $(u, v)$, there must be saturated chains $C_{1}, C_{2}$ both containing $u, v$ which agree outside of $(u, v)$, where $C_{1}$ is lexicographically smaller than $C_{2}$ on $(u, v)$ but where neither chain has any topological descents on $(u, v)$; let $C_{2}$ include $u=u_{1} \prec \cdots \prec u_{k}=v$. Then $C_{1}$ and $C_{2}$ must be related to each other in one of the following ways:

(1) The chains $C_{1}$ and $C_{2}$ both refine a pair of identical blocks $B_{1}, B_{2}$ of $u$ into pieces $\alpha_{1}, \ldots, \alpha_{r}, \beta_{1}, \beta_{2}$ in $v$ such that

(a) $\beta_{1}, \beta_{2}$ are the children of $B_{i}$ in $C_{i}$ with $\beta_{1}$ smaller than $\beta_{2}$ in our block order;

(b) $\alpha_{1}, \ldots, \alpha_{r}$ are the children of $B_{1}$ in $C_{2}$ and of $B_{2}$ in $C_{1}$ with $\alpha_{1}, \ldots, \alpha_{r}$ arranged in increasing order in our block order;

(c) $r>2$;

(d) $\alpha_{1}$ is larger than $\beta_{1}$ in our block order.

The blocks $B_{1}$ and $B_{2}$ are distinguished from each other by the fact that $B_{1}$ is refined before $B_{2}$ because it is farther to the left in the ordered partition $u$ (where the ordering of blocks in $u$ results from the choice of root that is common to $C_{1}$ and $C_{2}$ ). Both $C_{1}$ and $C_{2}$ refine the blocks of $u$ from left to right with the children increasing in length-lex order from left to right. Figure 6 gives an example with $u$ of $\operatorname{rank} 1, v$ of rank 4 , and the $C_{1}$ refinement represented by the top arrows and the $C_{2}$ refinement by

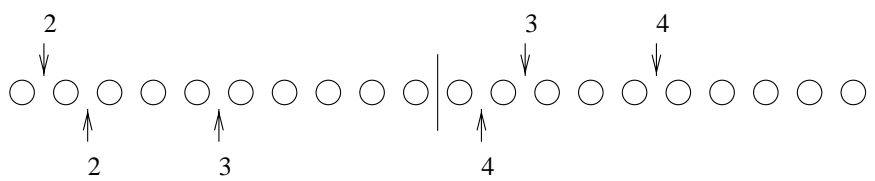

Figure 6 . An example of type 1 
the bottom arrows, both denoting ranks when bars are inserted in these positions.

(2) The chains $C_{1}$ and $C_{2}$ both split the same set of blocks of $u$ into the same set of smaller blocks in $v$ in two different ways by rearranging which children blocks belong to which types of parent. Ziegler constructed an interval of this type to show that the poset of partitions of an integer is not CohenMacaulay [26; he noted that $4=1+3=2+2 ; 7=2+5=1+6 ; 8=2+6=$ $3+5$, allowing the blocks $4,7,8$ to be split into the six pieces $1,3,2,2,5,6$ in two different ways, yielding two disjoint boolean algebras (and consequently two different saturated chains on the interval $(4|7| 8,2|2| 1|6| 3 \mid 5)$ both of which are free of topological descents), as depicted in Figure 7 Again we list $C_{1}$ above $C_{2}$ in the diagram.

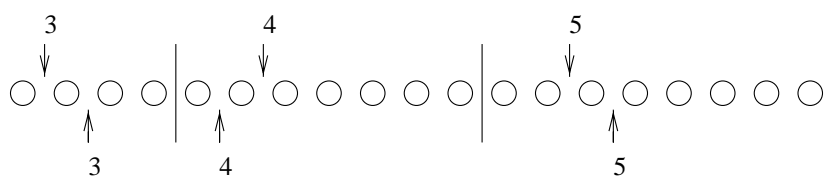

FIgURE 7 . An example of type 2

(3) Some block $B$ gets refined in $C_{1}$ but not in $C_{2}$ (or the reverse), because in the refinement in $C_{1}$, the block $B$ is a child of some larger block $B^{\prime}$ that also appears in $u$ and so the copy of $B$ in $u$ is refined and a new copy is recovered as a child of $B^{\prime}$ thereby appearing both in $u$ and $v$. Figure 8 gives an example for $u$ of rank $2, v$ of rank 5 with $B=1^{2}, B^{\prime}=1^{10}$.

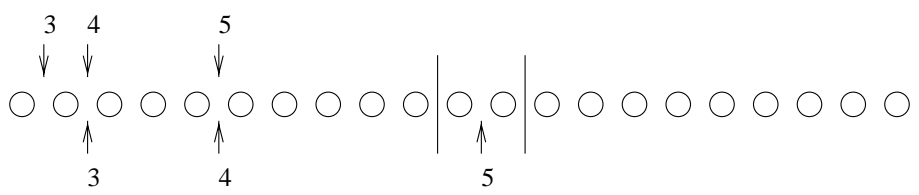

Figure 8. An example of type 3

It is not hard to see that these are the only basic possibilities given that $C_{2}$ cannot have any topological descents, so bars must be inserted left to right within $u$ with the children of each $u$-block increasing in length-lex order from left to right to obtain $v$. Case (1) is the only way the same types of blocks may be split into the same types of pieces, i.e. by rearranging which actual blocks in $u$ of a particular content are split which ways. Case (2) handles the possibility that the same set of blocks of $u$ gets split into the same set of smaller blocks in $v$, but with different types of blocks having different types of parents in $C_{1}$ and $C_{2}$. Then case (3) is the only way different actual blocks of $u$ could be refined with $C_{1}$ and $C_{2}$ both leading to the same set of blocks in $v$. Case (2) cannot happen for $\lambda$ of hook shape because it requires repetition of more than one type of letter in order for the same child blocks to come from more than one possible parent.

Every lower critical cell in a lexicographic discrete Morse function must have at least one nontrivial minimal skipped interval in $J$, and we find it convenient to pair lower critical cells based on their highest rank such interval. In each of the three 
cases, we will construct a partner cell by showing that for any particular highest rank nontrivial minimal skipped interval $(u, v)$ exactly one of the following two possibilities holds:

- There is a bar inserted to refine $v_{i}$ to get $v_{i+1}$ in $C_{2}$ within the interval $(u, v)$ whose insertion may be shifted to a rank coming after the interval $(u, v)$, yielding a lexicographically later saturated chain $C_{3}$ which has a skipped interval of height one smaller than $(u, v)$ and which has only topological descents after this skipped interval.

- The interval $(u, v)$ can be enlarged by shifting a single bar insertion into the interval from a later rank, yielding a lexicographically earlier saturated chain $C_{2}^{\prime}$ which has only topological descents after the interval that replaces $(u, v)$, so this has a (potentially critical) cell of dimension one smaller than the critical cell in $C_{2}$.

Either possibility guarantees the existence of a partner cell for each lower critical cell because the lower ranks of $C_{2}$ are unchanged, so that minimal skipped intervals in $I\left(C_{3}\right)$ (resp. $I\left(C_{2}^{\prime}\right)$ ) cover $C_{3}$ (resp. $C_{2}^{\prime}$ ). We will also show in a later section that there is a unique gradient path between these partner cells, as needed for cancellation. For the nontrivial minimal skipped intervals of types one and three, we will show that these partner cells are themselves critical, but it is not known whether this is always true for nontrivial minimal skipped intervals of type two.

5.3. Existence of partner critical cells. For each of the three types of nontrivial minimal skipped interval which may occur in $\Pi_{n} / S_{\lambda}$ as the highest rank such interval in some $e \sigma$, we describe how a partner cell $\tau$ is constructed and for types 1 and 3 we observe that this partner must always be critical. The constructions of partner cells generalize in an obvious way to mixtures of these "fundamental" types of nontrivial minimal skipped intervals, and mixtures of types 1 and 3 have critical partners for the same reasons that cells with intervals purely of type 1 or 3 have critical partners.

Let us begin with an example (where the nontrivial minimal skipped interval is of type 3), depicted in Figure 9.

$$
\begin{aligned}
& \mathrm{F}=\mathrm{h}|\mathrm{h}| \mathrm{i}|\mathrm{j}| \mathrm{k}|\mathrm{l}| \mathrm{h}|\mathrm{h}| \mathrm{g}|\mathrm{f}| \mathrm{e}|\mathrm{c}| \mathrm{d} \\
& \begin{array}{llllllllllll}
12 & 2 & 3 & 4 & 5 & 1 & 6 & 7 & 8 & 9 & 10 & 11
\end{array} \\
& \mathrm{G}=\mathrm{h}|\mathrm{h}| \mathrm{i}|\mathrm{j}| \mathrm{k}|\mathrm{l}| \mathrm{h}|\mathrm{h}| \mathrm{g}|\mathrm{f}| \mathrm{e}|\mathrm{c}| \mathrm{d} \\
& \begin{array}{llllllllllll}
12 & 2 & 3 & 4 & 11 & 1 & 5 & 6 & 7 & 8 & 9 & 10
\end{array}
\end{aligned}
$$

Figure 9. Two saturated chains whose critical cells are partners

Example 5.2. The saturated chain $F$ has nontrivial minimal skipped intervals with rank sets $\{1\},\{2\},\{3,4,5,6\},\{7\},\{8\},\{9\},\{10\},\{11\}$ yielding a critical cell $\sigma^{(6)}$ consisting of ranks $\{1,2,3,7,8,9,10\}$. The unique nontrivial minimal skipped interval in $F$ consists of rank set $\{3,4,5,6\}$ and results from the block $\{h, h\}$ also 
being contained in the block $\{h, h, g, f, e, c, d\}$ so that a lexicographically earlier saturated chain may sequentially insert bars into positions $6,2,1,3,4,5,8,9,10,11,7$ splitting the block $\{h, h\}$ into singletons immediately after its creation instead of later splitting off the two copies of $h$ which occur farther to the right. The lexicographically later saturated chain $G$ has nontrivial minimal skipped intervals with rank sets $\{1\},\{2\},\{3,4,5\},\{6\},\{7\},\{8\},\{9\},\{10\},\{11\}$ leading to a critical cell $\tau^{(7)}$ comprised of ranks $\{1,2,3,6,7,8,9,10,11\}$. The gradient path from $\tau$ to $\sigma$ begins by deleting the descent at rank 10 in $G$ and replacing it by an ascent; the gradient path continues by sequentially replacing ranks $9,8,7$ and 6 by ascents with the net effect of shifting the insertion of the bar between $k$ and $l$ from rank 11 to rank 5, increasing the height of the nontrivial minimal skipped interval by one and thereby giving a new critical cell of dimension one smaller.

We will always pair critical cells by shifting bars in or out of the last nontrivial minimal skipped interval. A later section will use permutation statistics to prove uniqueness of gradient paths, at which time we will revisit this example.

The first type of highest rank nontrivial minimal skipped interval for $e \sigma$ results from two identical blocks $B_{1}, B_{2}$ being refined in different ways, in which case $e \sigma$ refines $B_{1}$ in a lexicographically larger way than $\gamma$ does (letting $\gamma$ denote the lexicographically earliest saturated chain differing from $e \sigma$ only on this interval). Because the interval is nontrivial, either $e \sigma$ inserts multiple bars left to right in $B_{1}$ creating children from left to right which are nondecreasing before refining $B_{2}$, or there is at least one block to the right of $B_{1}$ and to the left of $B_{2}$ which also gets refined in the interval. In either case, let us refer to the series of bar insertions that are strictly within the skipped interval as $b_{1}, \ldots, b_{r}$. All higher ranks in $e \sigma$ must consist entirely of topological descents or skipped intervals which have height one after truncation. We defer discussion of truncation to the end of Theorem [5.2, and at this point note that it will not change our partnership construction because the descent to be shifted in or out of the interval occurs outside the interval at a higher rank than all such truncated intervals. Assuming there are only topological descents above the interval, the remainder of $e \sigma$ must entirely refine each of the remaining blocks with only topological descents and proceed from right to left among the blocks to be refined. We will have two quite different cases, depending on how the left child $L_{r}$ resulting from the refinement step $b_{r}$ compares to the left block $L_{R_{r}}$ obtained from the right child $R_{r}$ of the refinement $b_{r}$ when the block $R_{r}$ is first refined at some later point in the chain.

If $L_{r}>L_{R_{r}}$, then we pair $\sigma$ with a face $\tau$ such that $e \tau$ has the $b_{r}$ bar insertion shifted to after the skipped interval, increasing the number of minimal skipped intervals by 1 , so that $\operatorname{dim}(\tau)=\operatorname{dim}(\sigma)+1$ and $e \sigma<_{l e x} e \tau$. The bar insertion $b_{r}$ is shifted to the unique position later in the saturated chain which ensures there are still only topological descents after the skipped interval. Such a position exists precisely because $L_{r}>L_{R_{r}}$. One may check that $\tau$ is critical by observing that the skipped interval derived from the former highest rank nontrivial minimal skipped interval of $e \sigma$ is also minimal (of type 1).

When $L_{r} \leq L_{R_{r}}$, then we instead pair $\sigma$ with a critical cell $\rho$ such that $e \rho<_{l e x} e \sigma$ and $\operatorname{dim}(\tau)=\operatorname{dim}(\sigma)-1$. This is accomplished by shifting the bar insertion which creates the block $L_{R_{r}}$ into the minimal skipped interval increasing its height by one and decreasing the number of minimal skipped intervals by one. Because exactly one of the conditions $L_{r}>L_{R_{r}}$ or $L_{r} \leq L_{R_{r}}$ holds, we are ensured that there is 
not a series of more than two critical cells arising from shifting more and more bar insertions in or out of the interval, so there is no issue of multiple cells needing the same partner in this manner.

Now let us turn to the possibility that the last nontrivial minimal skipped interval is of type two, in which case the interval comes from refining a collection of blocks in two different ways, such as in Ziegler's example. When $\lambda$ is of hook shape, none of the critical cells may be of type two, since the blocks may only use one type of letter with repetition, but every letter appearing in the blocks to be refined must occur in at least two places; it is impossible to avoid topological ascents after the last nontrivial minimal skipped interval when using only the one type of letter which occurs with repetition. Let us nonetheless briefly discuss how to match critical cells $\tau$ whose last nontrivial minmimal skipped interval is of type two, in case this could be helpful for generalizing our result to all $\lambda$. What remains open is whether the partner cell for $\tau$ is itself critical. We pair the critical cell $\tau$ with a lexicographically earlier cell $\sigma$ by replacing the (topological) descent immediately following the last nontrivial minimal skipped interval by an ascent in a way so that this rank is incorporated into the last nontrivial minimal skipped interval. This is possible because the last block refined in the last nontrivial minimal skipped interval must have size at least three, because it is refineable in two different ways. Thus, this block needs further refinement after the nontrivial minimal skipped interval, so we insert such a refinement step into the interior of the skipped interval.

In the third case, there is a block of $u$ which is refined but then rearises as a child block later in one of the facets, while this same block is not refined at all in the other facet. This case proceeds identically to case one, so we omit the argument. The point is again to compare $L_{r}$ to $L_{R_{r}}$ to decide whether the partner critical cell should be lexicographically earlier or later.

5.4. Collections of inversions: A decreasing statistic. For each saturated chain, let us consider its restriction to those ranks above its last nontrivial minimal skipped interval. To each such restricted chain $v_{r} \prec \cdots \prec v_{n}$, where each $v_{k}$ has rank $k$, we associate a collection of rank inversions $(i, j)$ as follows: we include $(i, j)$ among the set of inversion pairs whenever either of the following conditions are met:

- The bar inserted at $\operatorname{rank} j$ (i.e. by the covering relation $v_{j-1} \prec v_{j}$ ) is farther to the right than the bar inserted at rank $i$

- The covering relations between ranks $i, i+1, \ldots, j-1, j$ insert bars left to right into a single block of $v_{i-1}$ in such a way that $L_{i}>L_{j}$.

Note that for the collection of inversions associated to a saturated chain to be exactly the inversions of some permutation, the following two facts are necessary:

(1) $(i, j),(j, k) \in S$ implies $(i, k) \in S$.

(2) $(i, k) \in S$ implies that $(i, j) \in S$ or $(j, k) \in S$ for each $i<j<k$.

The second condition implies that whenever $(i, k) \in S$ then there is also some $(j, j+1) \in S$ for $i \leq j<k$, enabling any permutation to be expressed as a product of adjacent transpositions. These two axioms do not hold for saturated chains in their entirety, but do hold when we restrict to the ranks above the last nontrivial minimal skipped interval, i.e. the ranks which are allowed to vary in gradient paths between pairs of critical cells to be cancelled. 
Remark 5.1. In an edge-labeling in which consecutive labels increase exactly where there are topological ascents, rank-inversions may be defined by comparing the labels at the respective ranks. This is not the situation in our chain-labeling for $\Pi_{n} / S_{\lambda}$.

5.5. Uniqueness of gradient paths. In this section, we check for critical cells $\tau^{(p+1)}, \sigma^{(p)}$ that were paired in Section 5.3 that the gradient path from $\tau$ to $\sigma$ is unique. This makes use of the statistic of the previous subsection. We return to the example of Section 5.3 immediately after proving the following theorem.

Theorem 5.2. If $\sigma, \tau$ are paired as in Section 5.3, then there is a unique gradient path from $\tau$ to $\sigma$.

Proof. Let $\tau^{(p+1)}$ and $\sigma^{(p)}$ be partner critical cells with $e \tau>_{\text {lex }} e \sigma$. Consider the gradient path from $\tau$ to $\sigma$ as given in Section 5.3. If $e \tau$ and $e \sigma$ agree on ranks $1,2, \ldots, i$, then gradient paths from $\tau$ to $\sigma$ may never delete elements of rank less than or equal to $i$. This is because any such deletion $\tau_{i} \rightarrow \sigma_{i}$ would leave a minimal skipped interval of $e \tau$ uncovered so that $e \sigma_{i}<_{l e x} e \sigma$, implying $f\left(\sigma_{i}\right)<f(\sigma)$. This would make it impossible to complete the gradient path to $\sigma$ with $f$ decreasing at each step. Thus, we need only consider gradient path steps deleting ranks above the last nontrivial minimal skipped interval, since $\sigma$ and $\tau$ agree up through the first rank in this interval.

To this end, we shall use inversions to prove uniqueness. Let us temporarily assume that there are only topological descents after the last nontrivial skipped interval. Later we will consider the possibility of nontrivial minimal skipped intervals which are reduced to height one by truncation. Given a gradient path from $\tau$ to $\sigma$, each step in a gradient path eliminates a single inversion since the downward step $\tau_{i} \rightarrow \sigma_{i}$ must uncover a topological descent yielding $e \sigma_{i}<_{l e x} e \tau_{i}$ so that our matching forces $\tau_{i+1}$ to have this rank, which we call $r$, reinserted with a lexicographically earlier topological ascent eliminating a single rank inversion $(r, r+1)$. Denote by $d_{i_{1}} \circ \cdots \circ d_{i_{k}}$ a gradient path which deletes rank $i_{j}$ at the step $\tau_{j} \rightarrow \sigma_{j}$ for each $j$. The inversion set of $\tau$ is then the inversion set of $\sigma$ together with the set of inversions found in the permutation $s_{i_{1}} \circ \cdots \circ s_{i_{k}}$, where $s_{i}$ denotes the adjacent transposition $(i, i+1)$ swapping $i$ and $i+1$. Every other gradient path from $\tau$ to $\sigma$ must similarly give rise to another reduced expression for this same permutation since every gradient path from $\tau$ to $\sigma$ must eliminate the same set of inversions.

Furthermore, we need for the indices in the word $d_{i_{1}} \circ \cdots \circ d_{i_{k}}$ to be monotonically decreasing so that a downward step in a gradient path always leads to a vertex that is matched with one above rather than below it; the cyclic shifts which arise from shifting a single bar insertion into the last nontrivial minimal skipped interval from a later rank meet this requirement. Note that the downward step $d_{i}$ eliminates an inversion at rank $i$ and the upward step via the matching replaces this with a lexicographically earlier ascent at $\operatorname{rank} i$, so then applying $d_{j}$ immediately after $d_{i}$ for $j>i$ would lead downward to a face $\sigma_{k}$ which includes rank $i$ as an ascent, hence as a cone point in the intersection of $e \sigma_{k}$ with earlier facets, so that $\sigma_{k}$ is the top of a matching edge in an earlier fibre, making it impossible for us to continue our gradient path with an upward step from $\sigma_{k}$. We have paired critical cells $\sigma^{(p)}, \tau^{(p+1)}$ so that the permutation associated to the gradient path from $\tau$ to $\sigma$ is a cyclic shift $s_{j} s_{j-1} \cdots s_{i+1} s_{i}$ with decreasing indices which has a unique reduced expression, as desired. 
Now let us deal with the possibility of nontrivial skipped intervals in $I(e \sigma)$ or $I(e \tau)$ which become trivial in $J$ and which come after the last nontrivial interval of $I$ that remains nontrivial in $J$. (Let us call this last interval $\gamma$.) Notice first that these must all occur consecutively immediately after $\gamma$, and after this we have only topological descents. The bars inserted immediately after $\gamma$ up until the topological descents all must be inserted left to right to avoid descents. The bar insertion which is shifted into $\gamma$ in the gradient path from $\tau$ to $\sigma$ must be farther to the left than all these intermediate bar insertions so that there are rank inversions in $\tau$ between the bar insertion being shifted and the bar insertions in the truncated nontrivial intervals. This means the bar insertion of $\gamma$ shifts across these truncated intervals just as it shifts across descents.

Now consider the critical cells $\sigma$ and $\tau$ of Example 5.2 The facets $F$ and $G$ agreed up through rank 4 which means that only ranks of 5 or greater may be deleted in a gradient path from $\tau$ to $\sigma$. The restriction of $F$ to ranks above its last nontrivial minimal skipped interval only has a single rank inversion $(11,12)$ while the restriction of $G$ has inversions $(11,12),(10,11),(9,11),(8,11),(7,11),(6,11)$ so that the extra inversions of $G$ are exactly the inversions of the permutation $s_{10} \circ$ $s_{9} \circ s_{8} \circ s_{7} \circ s_{6} \circ s_{5}=(6,7,8,9,10,11)$. Any gradient path $d_{i_{1}} \circ \cdots \circ d_{i_{k}}$ from $\tau$ to $\sigma$ would need to remove from $G$ the same inversions that are present in the permutation $s_{i_{1}} \circ \cdots \circ s_{i_{k}}$ to be a legitimate gradient path, but since the cyclic shift $(6,7,8,9,10,11)$ has a unique reduced expression there cannot be any other gradient paths from $\tau$ to $\sigma$.

5.6. An injective partnership map on lower critical cells. Section 5.3 proved that for every lower critical cell there exists at least one critical cell with which it could be cancelled. Now we will choose how to pair critical cells to be cancelled so that (1) no two critical cells are paired with the same critical cell and (2) the pairing is compatible with lexicographic order (i.e. there is never a critical cell $\gamma$ such that $\sigma<_{\text {lex }} \gamma<_{\text {lex }} \tau$ for $\sigma, \tau$ a pair of critical cells we intend to cancel with each other). Thus each lower critical cell is paired with another critical cell such that there is a unique gradient path from one to the other both in the lexicographic discrete Morse function and also in the subsequent discrete Morse functions derived from it by reversing the unique gradient paths between lexicographically earlier pairs of matched critical cells. Our pairing of critical cells may involve some of the topdimensional critical cells, but certainly does not need to involve all of them since our goal is only to cancel all the lower critical cells.

Recall that for nontrivial minimal skipped intervals of types 1 and 3, we checked that given a critical cell $\sigma$, then exactly one of the cells obtained by shifting a bar into or out of the interval will be critical, so we do not need to be concerned with the possibility that two different critical cells would both be paired with $\sigma$ in this fashion. In either case, the point was that a bar may be inserted in the last nontrivial skipped interval of $\tau$ (or some subsequent descent) to yield a facet with a critical cell if and only the last bar insertion in the last nontrivial minimal skipped interval (or in the later topological descent) may not be extracted. The if and only if statement is determined by whether the block created later immediately to the right of the bar to be extracted is bigger or smaller than the block immediately to the left of this bar (created when this bar is inserted). Thus we can be sure there 
is exactly one critical cell that may be obtained from $\sigma$ based on the last nontrivial minimal skipped interval in $\sigma$.

Now let us show that whenever two critical cells $\sigma$ and $\tau$ are paired as in Section 5.3 but there is another critical cell $\sigma^{\prime}$ such that $\sigma<_{\text {lex }} \sigma^{\prime}<_{\text {lex }} \tau$, then $\sigma^{\prime}$ may be paired with either $\sigma$ or $\tau$; furthermore, when $\sigma \sim \sigma^{\prime}$ (resp. $\tau \sim \sigma^{\prime}$ ), then there will also exist a critical cell $\rho$ which may be paired with $\tau$ (resp. with $\sigma$ ). The critical cell $\rho$ exists by virtue of the structure of $\sigma$ after its last nontrivial skipped interval. We always pair each critical cell with the partner which agrees with it up to the highest rank; the point is that if there is some cell agreeing with $\sigma$ up to a higher rank than $\tau$ has, then the similarity between $\sigma$ and $\tau$ after this rank ensures there is also a similar partner critical cell for $\tau$.

Example 5.3. Figure 10 gives a saturated chain which has two nontrivial minimal skipped intervals, each skipping only two ranks. These intervals go from rank 4 to 7 and from rank 14 to 17 . The critical cell $\sigma$ of this facet is paired with a lexicographically later critical cell $\tau$ which has the second nontrivial skipped interval of $e \sigma$ replaced by a skipped interval of height one, which means Section 5.3 provides a possible partner for $\tau$ by exhibiting a different critical cell $\rho$ which could be paired with $\tau$ (based on the only nontrivial minimal skipped interval of $\tau$, i.e. the first of two nontrivial minimal skipped intervals for $\sigma$ ). One may obtain a partner critical cell for $\rho$, as needed, by replacing a topological descent in $e \rho$ with a nontrivial minimal skipped interval of height 2 just as is done in converting from $\sigma$ to $\tau$.

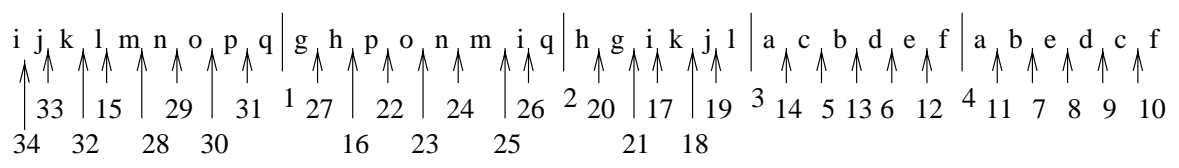

Figure 10. A saturated chain $e \sigma$ to be paired with $\rho$

Suppose the nontrivial minimal skipped interval of highest rank in $e \sigma^{\prime}$ is an interval skipping two ranks. Then $\sigma^{\prime}$ is paired with a critical cell $\sigma$ which instead skips either one or three ranks. In the case of skipping one rank, this is no longer nontrivial, so $\sigma$ will be paired in Section 5.3 based on an earlier nontrivial minimal skipped interval with a different critical cell $\rho$. However, $\sigma^{\prime}$ agrees with $\sigma$ to a later rank, so we choose $\sigma \sim \sigma^{\prime}$. Because $\sigma$ is related to $\rho$ by the shifting of a single bar insertion to after the nontrivial minimal skipped interval, $\rho$ possesses the same later structure that gave rise to a partner critical cell for $\sigma$, and this structure will likewise give rise to a partner $\rho^{\prime}$ for $\rho$.

5.7. Möbius functions for $\Pi_{n} / S_{\lambda}$. Now that we have proven the homotopy type for $\lambda$ of hook shape is a wedge of spheres concentrated in top dimension, let us conclude with an example of how this may be helpful for computing Möbius functions.

Proposition 5.1. Let $\lambda$ be of hook shape. Then $\mu_{P}(\hat{0}, \hat{1})=0$ if and only if $\lambda_{1}>$ $3(l(\lambda)-1)$ where $l(\lambda)$ is the length of $\lambda$, i.e. the number of nonempty blocks.

Proof. We claim that our lexicographic discrete Morse function for $\Pi_{n} / S_{\lambda}$ has uncancelled top-dimensional critical cells if and only if $\lambda_{1} \leq 3[l(\lambda)-1]$. Let $\lambda=$ $\left(m, 1^{k}\right)$. Let us represent elements of $\Pi_{n} / S_{\lambda}$ by partitions of $\left\{a^{m}, b_{1}, b_{2}, \ldots, b_{k}\right\}$. When $m=3, k=1$, then there is a critical cell in the facet $\left.\left.\left.a\right|_{3} a\right|_{1} a\right|_{2} b_{i}$ that is not 
cancelled by any lower critical cell. More generally, when $m=3 k$, then there is a top-dimensional critical cell that is not cancelled in the facet which begins by inserting bars left to right to form the partition

$$
a a a b_{k}\left|a a a b_{k-1}\right| \cdots \mid a a a b_{1}
$$

and then refines blocks right to left as in the $a^{3} b_{i}$ example; this critical cell also is not cancelled by any lower one. Similar examples may be constructed for $m<3 k$. On the other hand, there are no critical cells when $m=4, k=1$ (and similarly for $k=1, m>4)$. Any saturated chain with $m>3 k$ will have a topological ascent (and hence no top-dimensional critical cells) because each refinement preserves the property $m>3 k$ for some component (by the pigeonhole principle), eventually reducing to the case $k=1, m \geq 4$.

Since all lower critical cells are cancelled in our proof regarding the homotopy type for $\lambda$ of hook shape, the nonexistence of top-dimensional critical cells for $m>3 k$ implies the Möbius function $\mu_{P}(\hat{0}, \hat{1})$ must be 0 for such $m, k$.

5.8. Generalization to arbitrary multisets. The reason our proof concerning the homotopy type of $\Delta\left(\Pi_{n} / S_{\lambda}\right)$ does not as it stands apply to all $\lambda$ is that we do not know whether every lower critical cell is paired with a cell which is also critical. More specifically, the partner cells for critical cells based on nontrivial minimal skipped intervals of type 2 may or may not themselves be critical. This is because they each have nontrivial skipped intervals of height one larger than the type 2 interval they are replacing, but we do not know whether these new intervals are always minimal skipped intervals. Thus, we do not know for sure that the saturated chains contributing these cells are covered by minimal skipped intervals. If this were verified, then our proof would apply to all $\lambda$.

One way to prove that these intervals must be minimal would be to show that a certain puzzle on multisets has no solutions. One approach might be to specialize to integer partitions and prove that the resulting puzzle already has no solutions. If (conversely) there were a solution in integer partitions, one could probably translate this to a solution in multisets with enough distinct letters that one could find a lower critical cell that cannot be cancelled by any other critical cell, so this would probably yield a poset that is not homotopy equivalent to a wedge of spheres of top dimension.

The puzzle is as follows: is there a set of integers $n_{1}, \ldots, n_{k}$ for $k \geq 3$ and a more refined set $\left\{b_{i, j} \mid 1 \leq i \leq k, 1 \leq j \leq a_{i}\right\}$ of integers so that:

(1) $n_{i}=b_{i, 1}+\cdots+b_{i, a_{i}}$ for $i<k$ with each $a_{i} \geq 2$.

(2) $n_{k}=b_{k, 1}+b_{k, 2}=c_{k, 1}+c_{k, 2}$ for some $b_{k, 1}<b_{k, 2}, c_{k, 1}<c_{k, 2}$ with $b_{k, 1}>c_{k, 1}$.

(3) The collection of numbers $\left\{b_{r, s} \mid s<k\right\} \cup\left\{c_{k, 1}, c_{k, 2}\right\}$ may be partitioned into blocks $B_{1}, \ldots, B_{k}$ so that the sum of the numbers in block $B_{i}$ is $n_{i}$, and the smallest block assigned to $B_{i}$, denoted $b_{i, 1}^{\prime}$, is at least as small as $b_{i, 1}$. If $b_{i, 1}^{\prime}=b_{i, 1}$, then list the blocks $b_{i, j}^{\prime}$ assigned to $B_{i}$ in increasing order, and we require that the first $b_{i, j}^{\prime}$ to differ from the corresponding block $b_{i, j}$ be smaller than $b_{i, j}$.

We are not sure whether the integer partitions puzzle has solutions, and suggest it as a possible way of getting at the homotopy type of $\Delta\left(\Pi_{n} / S_{\lambda}\right)$ for general $\lambda$. It might even suffice to show that there are no solutions when the following additional 
requirement is also imposed on the puzzle: that the numbers in the set $\left\{b_{r, s} \mid s \leq k\right\}$ which are larger than one are all distinct. Likewise, require the numbers in the set $\left\{b_{r, s} \mid s<k\right\} \cup\left\{c_{k, 1}, c_{k, 2}\right\}$ which are larger than one all to be distinct.

\section{ACKNOWLEDGMENTS}

The authors thank Xun Dong, Art Duval and Robin Forman for proofreading portions of our paper and providing valuable feedback.

\section{REFERENCES}

[1] E. Babson, A. Björner, S. Linusson, J. Shareshian and V. Welker, Complexes of not iconnected graphs, Topology 38 (1999), 271-299. MR1660341(2000a:57001)

[2] E. Babson and D. Kozlov, Group actions on posets, To appear in J. Algebra.

[3] L. Billera and G. Hetyei, Linear inequalities for flags in graded partially ordered sets, J. Combin. Theory Ser. A 89 (2000), 77-104. MR1736134 (2001m:52011)

[4] A. Bjorner, Shellable and Cohen-Macaulay partially ordered sets, Trans. Amer. Math. Soc. 260 (1980), no. 1, 159-183. MR0570784 (81i:06001)

[5] A. Björner, Posets, regular CW complexes and Bruhat order, European J. Combin. 5 (1984), no. 1, 7-16. MR0746039 (86e:06002)

[6] A. Björner, Topological Methods, in Handbook of Combinatorics (R. Graham, M. Grötschel and L. Lovasz, eds.), North-Holland, Amsterdam, 1993. MR1373690 (96m:52012)

[7] A. Björner and M. Wachs, Bruhat order of Coxeter groups and shellability, Adv. in Math. 43 (1982), 87-100. MR0644668 (83i:20043)

[8] A. Bjorner and M. Wachs, On lexicographically shellable posets, Trans. Amer. Math. Soc. 277 (1983), no. 1, 323-341. MR0690055 (84f:06004)

[9] A. Björner and M. Wachs, Nonpure shellable complexes and posets I, Trans. Amer. Math. Soc. 348 (1996), 1299-1327. MR1333388 (96i:06008)

[10] A. Björner and M. Wachs, Nonpure shellable complexes and posets II, Trans. Amer. Math. Soc. 349 (1997), 3945-3975. MR1401765 (98b:06008)

[11] M. Chari, On discrete Morse functions and combinatorial decompositions, Discrete Math 217 (2000), no. 1-3, 101-113. MR1766262 (2001g:52016)

[12] J. Folkman, The homology groups of a lattice, J. Math. Mech. 15 (1966), 631-636. MR.0188116 (32:5557)

[13] R. Forman, Morse theory for cell complexes, Adv. Math. 134 (1998), 90-145. MR1612391 (99b:57050)

[14] P. Hersh, Lexicographic shellability for balanced complexes, J. Algebraic Combin. 17 (2003), no. 3, 225-254. MF 2001670 (2004f:05190)

[15] P. Hersh, On optimizing discrete Morse functions, Preprint 2003.

[16] P. Hersh and R. Kleinberg, The refinement complex of the poset of partitions of a multiset, In preparation.

[17] P. Hersh and V. Welker, Gröbner basis degree bounds on $\operatorname{Tor}^{k[\Lambda]}(k, k)$, To appear in Proceedings of the AMS-IMS-SIAM Joint Summer Research Conference on Integer Points in Polyhedra.

[18] D. Kozlov, General lexicographic shellability and orbit arrangements, Ann. of Comb. 1 (1997), no. 1, 67-90. MR1474801 (98h:52023)

[19] D. Kozlov, Complexes of directed trees, J. Combin. Theory Ser A 88 (1999), no. 1, 112-122. MR 1713484 (2000j:05036)

[20] G-C. Rota, On the foundations of combinatorial theory I: Theory of Möbius functions, Z. Wahrsch. 2 (1964), 340-368. MR0174487 (30:4688)

[21] J. Shareshian, On the shellability of the order complex of the subgroup lattice of a finite group, Trans. Amer. Math. Soc. 353 (2001), no. 7, 2689-2703. MR1828468 (2002k:06006)

[22] R. Stanley, Supersolvable lattices, Algebra Universalis 2 (1972), 197-217. MR0309815 (46:8920)

[23] R. Stanley, Enumerative Combinatorics, vol. I. Wadsworth and Brooks/Cole, Pacific Grove, CA, 1986; second printing, Cambridge University Press, Cambridge/New York, 1997. MR:0847717 (87j:05003) 
[24] R. Stanley, Combinatorics and Commutative Algebra, second ed., Birkhäuser, Boston, 1996. MR.1453579(98h:05001)

[25] V. Welker, Direct sum decompositions of matroids and exponential structures, J. Combin. Theory Ser. B 63 (1995), no. 2, 222-244. MR1320168 (96e:05042)

[26] G. Ziegler, On the poset of partitions of an integer, J. Combin. Theory Ser. A 42 (1986), no. 2, 215-222. MR0847552 (87k:06009)

Department of Mathematics, Box 354350, University of Washington, Seattle, WashINGTON 98195

E-mail address: babson@math.washington.edu

Department of Mathematics, Box 354350, University of Washington, Seattle, WashINGTON 98195

Current address: The Mathematical Sciences Research Institute, 17 Gauss Way, Berkeley, California 94720-5070

E-mail address: phersh@msri.org 